\title{
ANALYTIC FUNCTIONS IN BANACH SPACES
}

\author{
E. F. WhitTLESEY
}

Introduction. In [1] and [5] differential calculus is developed for (real or complex) Banach spaces in a dimension-free manner with scarcely more ado than in the 1-dimensional case. Our purpose is to demonstrate that a similar treatment, without reference to dimension, is available for analytic functions (real or complex Banach spaces) with equal simplicity. In case the domain or range is $R^{n}$ or $C^{n}$ the theory comprehends the classic one; but we need no polycylinders.

We shall not write a treatise, but merely set up the basic definitions, derive a few classic theorems, and mention one or two points of caution. The reader will find further similar generalizations and details easy (not the fundamental theorem of algebra, to be sure) using some of the standard techniques of our references. We suppose more or less familiarity with those references and we shall use the notation of [1] and [5]. $\mathrm{Cl} A$ will denote the closure of $A$; Int $A$ the interior of $A . C^{n}$ or $C^{n}(D: Y), n=1, \cdots, \infty, a$, denotes the class of maps with domain $D$ and image in $Y$ of continuous differentiability class $n=1, \cdots, \infty$, and $n=a$ means analytic. $X$ and $Y$ denote Banach spaces, $D$ an open set in $X . N_{r}(x)$ stands for the open ball, center $x$, radius $r$. A diffeomorphism $f: D \rightarrow f(D) \subset Y$ is a homeomorphism of class $C^{n}$, with $f^{\prime}(x)$ a topological isomorphism for each $x$ (in $D$ ). We shall obtain, among other results, the inverse and implicit function theorems for analytic functions without reference to dimension or scalar field.

Let $x, x_{1}, \cdots, x_{n} \in X$ and $a_{n}$ a continuous, symmetric, $n$-linear map of $X^{n}$ into $Y$, i.e. $a_{n} \in L_{s}^{n}(X: Y)$. In $a_{n}\left(x_{1}, \cdots, x_{n}\right)$, we can restrict the $x_{i}$ to the diagonal, i.e. to be equal; $a_{n}(x, \cdots, x)$ will be abbreviated $a_{n} x^{n}$. A power series in $x$ with values in $Y$ is a series of the form $\sum_{n=0}^{\infty} a_{n} x^{n}$, where $a_{0}$ is a point of $Y$. A power series with only a finite number of terms is a polynomial, $\sum_{k=0}^{n} a_{k} x^{k}$. If $a_{n} \neq 0$, then the polynomial has degree $n$. (The function $a_{n}$ is 0 iff it vanishes identically on the diagonal, since the $n$th derivative of $a_{n} x^{n}$ is $n ! a_{n}$.)

THEOREM. If $\sum_{0}^{\infty} a_{n} x^{n}$ converges, or if the terms are only bounded, and if $z=t x, t$ scalar, $|t|<1$, then $\sum a_{n} z^{n}$ converges absolutely.

PRoof. The terms $\left|a_{n} x^{n}\right|$ are bounded, say $\leqq K$. Then $\left|a_{n} z^{n}\right|$ $=\left|a_{n} x^{n}\right||t|^{n} \leqq K|t|^{n}$. Whence absolute convergence. q.e.d.

Received by the editors July 1, 1964. 
Consequently, for each direction $x$, if the series converges in that direction at all, there is a real number $r_{x}>0$, such that if $z=t x$, $t$ scalar, $\sum a_{n} z^{n}$ converges absolutely for $|z|<r_{x}$, and diverges for $|z|>r_{x}$. Of course, $\sum a_{n} x^{n}$ converges for $x=0$.

We consider now the case that $\sum\left|a_{n}\right|\left|x_{0}\right|^{n}$ converge for some $x_{0} \neq 0$. Then, of course, $\sum a_{n} x^{n}$ converges absolutely and uniformly for $|x| \leqq\left|x_{0}\right|$, and the sum is a continuous function. We say a function $f(x)$, defined in a neighborhood of a point $x_{0}$, is analytic at $x_{0}$ if it coincides with a convergent power series $\sum_{0}^{\infty} a_{n}\left(x-x_{0}\right)^{n}$ such that $\sum_{0}^{\infty}\left|a_{n}\right|\left|x-x_{0}\right|^{n}$ converges for $x$ near $x_{0} . f$ is analytic on an open set $D$ if it is analytic at each point of $D$.

If one differentiates $a_{n} x^{n}$ one obtains $a_{n}^{\prime}\left(x^{n}\right)(h)=\sum_{k=0}^{n-1} a_{n}\left(x^{k} h x^{n-k-1}\right)$ $=n a_{n} x^{n-1} h$, since $a_{n}$ is symmetric. Thus $a_{n}^{\prime} x^{n}=n a_{n} x^{n-1}$ (last argument is omitted). Similarly for higher derivatives: $a_{n}^{\prime \prime} x^{n}$ $=n(n-1) a_{n} x^{n-2}, \cdots, \quad a_{n}^{(n)} x^{n}=n ! a_{n}, \quad$ and $a_{n}^{(k)} x^{n}=0, \quad k>n$. Now $a_{n}^{\prime}\left(x^{n}\right)(h)=n a_{n} x^{n-1} h$ is a specialization to the diagonal of the map $n a_{n}\left(x_{1}, \cdots, x_{n-1}, h\right)$ which is $(n-1)$-linear, continuous, symmetric in the $x_{i}$ 's. We have $\left|n a_{n}\left(x_{1}, \cdots, x_{n-1}, h\right)\right| \leqq n\left|a_{n}\right| \prod_{1}^{n-1}\left|x_{i}\right||h|$. Thus $\left|a_{n}^{\prime}\right| \leqq n\left|a_{n}\right|, a_{n}^{\prime}$ being that $(n-1)$-linear map in the $x_{i}^{\prime}$ 's which is linear in $h$. Similarly, $\left|a_{n}^{(k)}\right| \leqq n(n-1) \cdots(n-k+1)\left|a_{n}\right|, 1 \leqq k \leqq n$.

THEOREM. If $f(x)=\sum_{0}^{\infty} a_{n}\left(x-x_{0}\right)^{n}$ has radius $r>0$, then $f(x)$ is analytic at any point $x_{1}$ in $N_{r}\left(x_{0}\right), f(x)=\sum_{k=0}^{\infty} b_{k}\left(x-x_{1}\right)^{k}$, and the radius there is $\geqq r-\left|x_{1}-x_{0}\right|$, and

$$
b_{k}=\sum_{n=0}^{\infty}\left(\begin{array}{c}
n+k \\
n
\end{array}\right) a_{n+k}\left(x_{1}-x_{0}\right)^{n} .
$$

Proof. Essentially the same as in the real case.

Theorem. If $\sum_{0}^{\infty}\left|a_{n}\right|\left|x-x_{0}\right|^{n}$ converges for $\left|x-x_{0}\right|<r$, then the function $f(x)=\sum_{0}^{\infty} a_{n}\left(x-x_{0}\right)^{n}$ has a derivative at any point $x$ such that $\left|x-x_{0}\right|<r$, which is $f^{\prime}(x)=\sum_{1}^{\infty} n a_{n}\left(x-x_{0}\right)^{n-1}$, and the new series converges for $\left|x-x_{0}\right|<r$, as does $\sum_{1}^{\infty} n\left|a_{n}\right|\left|x-x_{0}\right|^{n-1}$.

Proof. The real series $\sum\left|a_{n}\right| t^{n}$ and $\sum n\left|a_{n}\right| t^{n-1}$ have the same radius. Obviously, $f^{\prime}\left(x_{0}\right)=a_{1}$. Expand $f(x)$ about $x_{1}$, where $\left|x_{1}-x_{0}\right|$ $<r$, by the preceding, get $f(x)=\sum_{k=0}^{\infty} b_{k}\left(x-x_{1}\right)^{k}$. But, by the preceding remark, $f^{\prime}\left(x_{1}\right)=b_{1}$, and

$$
b_{1}=\sum_{n=0}^{\infty}\left(\begin{array}{c}
n+1 \\
n
\end{array}\right) a_{n+1}\left(x_{1}-x_{0}\right)^{n}=\sum_{n=1}^{\infty} n a_{n}\left(x_{1}-x_{0}\right)^{n-1} \text {. q.e.d. }
$$

So analyticity implies of class $C^{\infty}$, and observe that $f\left(x_{0}\right)=a_{0}, \cdots$, $f^{(n)}\left(x_{0}\right)=n ! a_{n}$. We call the radius of convergence of the real series 
$\sum_{0}^{\infty}\left|a_{n}\right| t^{n}$ the radius of the series $\sum a_{n}\left(x-x_{0}\right)^{n}$. We shall not be concerned here with points outside that sphere of convergence where the series may converge. (See [3, Chapter XXVI].) If the domain $X$ happens to be $R^{n}$ or $C^{n}(=C \times \cdots \times C, n$ factors, $C$ the complex field), and if one uses the multilinearity of the coefficients, and the standard base for $R^{n}$ or $C^{n}$, one obtains the classic form of a power series in several real or complex variables (values complex, or Banachvalued, whatever $Y$ may be). We leave it to the reader to verify that if $\sum\left|a_{n}\right||x|^{n}$ converges in a neighborhood of the origin, in $R^{n}$ or $C^{n}$ then the series obtained in "several variables" from $\sum a_{n} X^{n}$ which corresponds, also converges, absolutely, in a polycylinder about the origin, and conversely; (we do not assert that the domains coincide; we assert that convergence of one near the origin guarantees that the other converges in a neighborhood of the origin). The proof is elementary and requires only the definition of norm of a multilinear function. In particular, the computation shows that the sense of entire function (having infinite radius) is the same for both.

THEOREM. If an analytic function, $f(x)=\sum_{0}^{\infty} a_{n}\left(x-x_{0}\right)^{n}$ vanishes near $x_{0}$, then $a_{n}=0$, for all $n$.

Proof. For simplicity, let $x_{0}=0$. By continuity, $a_{0}=0$. Then $-a_{1} x=\sum_{k=1}^{\infty} a_{k+1} x^{k+1}$ near the origin. Replace $x$ by $t x, t$ scalar, $0<|t|<1$, obtain $-a_{1} x=\sum_{k=1}^{\infty} a_{k+1} x^{k+1} t^{k}$. The right side $\rightarrow 0$ with $t$. Hence $a_{1} x=0$. But $x$ is arbitrary near 0 . So $a_{1} x=0$, all $x$, i.e. $a_{1}=0$. Similarly, $a_{2} x^{2}=0$, all $x$, so $a_{2}=0$. Continue inductively. q.e.d. (Note that just the convergence of $\sum a_{n} x^{n}$ is needed, not absolute, or that of $\sum\left|a_{n}\right||x|^{n}$.)

Corollary (IDentity Theorem for Power Series). If two analytic functions, $f(x)=\sum a_{n}\left(x-x_{0}\right)^{n}$ and $g(x)=\sum b_{n}\left(x-x_{0}\right)^{n}$ coincide near $x_{0}$, then $a_{n}=b_{n}$ all $n$. (Observe that all that is needed is that two series coincide, on every line through $x_{0}$ (real or complex), on an infinite point set accumulating at $x_{0}$.)

ThEOREM. The composition of analytic functions is analytic. Precisely: if $g: D \rightarrow E$, and $f: E \rightarrow Z$ are analytic, $D$ open in $X, E$ open in $Y$, then $f g$ is analytic. In fact, if $N_{r}\left(x_{0}\right) \subset D$ and $g(x)=\sum a_{n}\left(x-x_{0}\right)^{n}$ has radius $\geqq r$, and $N_{\text {s }}\left(y_{0}\right) \subset E$, and $f(y)=\sum b_{m}\left(y-y_{0}\right)^{m}$ has radius $\geqq s$, and $g N_{r}\left(x_{0}\right) \subset N_{s}\left(y_{0}\right)$ and $g\left(x_{0}\right)=y_{0}$, then the series expansion $f g(x)$ $=\sum_{0}^{\infty} c_{k}\left(x-x_{0}\right)^{k}$ is obtainable from that for $f(y)$ and $g(x)$ by substitution of that for $g(x)$ for $y$, i.e. of $g(x)-g\left(x_{0}\right)$ for $y-y_{0}$, and collection of terms of common degree, and the radius of the new series in $x-x_{0}$ is at least $r$. 
Proof. The proof is similar to the scalar case. The constant term is $c_{0}=b_{0}$. The first degree term is $c_{1}\left(x-x_{0}\right)=b_{1} \circ a_{1}\left(x-x_{0}\right)$. The second is $c_{2}\left(x-x_{0}\right)^{2}=b_{2}\left(a_{1}\left(x-x_{0}\right), a_{1}\left(x-x_{0}\right)\right)+b_{1} \circ a_{2}\left(x-x_{0}\right)^{2}$, which we write $\left(b_{2} a_{1}^{2}+b_{1} a_{2}\right)\left(x-x_{0}\right)^{2}$, etc.

Theorem. If $f: D \rightarrow \prod_{1}^{n} Y_{i}$, then $f$ is analytic iff all its coordinates areand, then, if $f(x)=\sum_{m} a_{m}\left(x-x_{0}\right)^{m}, f_{i}(x)=p_{i} f(x)=\sum_{m} p_{i} a_{m}\left(x-x_{0}\right)^{m}$ $=\sum_{m} a_{m i}\left(x-x_{0}\right)^{m}$ and $f(x)=\sum_{m=0}^{\infty}\left(\sum_{i=1}^{n} a_{m i}\right)\left(x-x_{0}\right)^{m}$.

The proof is trivial, since projection, $p_{i}$, is continuous linear.

THEOREM. If $f$ and $g$ are analytic on an open convex set $D$ and if they coincide on a nonempty open subset $S$ of $D$, then they coincide on $D$.

Proof. Let $x$ be in $S, y$ in $D$, and $h(t)=f(x+t(y-x))-g(x+t(y-x))$, $t$ real. This is an analytic function of $t$ in a neighborhood of $[0,1]$. $h(0)=0$, and $h$ is analytic near 0 , so vanishes on an interval $[0, r)$ in $[0,1]$, so, by continuity, in $[0, r]$. Let $b=\sup$ such $r$. Suppose $b<1$. By analyticity at $b$, since $h$ is 0 just below $b, h$ is 0 just above $b$, i.e. in a neighborhood of $b$. Contradiction. So $b=1$.

Corollary. If $f(x)=\sum a_{n}\left(x-x_{0}\right)^{n}$ and $g(x)=\sum b_{n}\left(x-x_{0}\right)^{n}$ are analytic for $\left|x-x_{0}\right|<r$, and if they coincide on an open subset of $N_{r}\left(x_{0}\right)$, then $a_{n}=b_{n}$, for every $n$.

COROLLARY. If $f$ and $g$ are analytic in a region $D$ and coincide on $a$ nonempty open subset of $D$, then they coincide throughout $D$.

Proof. Let $C$ be the domain of coincidence. Int $C$ is not empty and is open in $D$. If $x_{0}$ is a point of closure of Int $C$ in $D$, then, since $f$ and $g$ are analytic at $x_{0}$, hence have expansions about $x_{0}$ with some common radius $r>0$, and since they coincide on an open subset of $N_{r}\left(x_{0}\right)$, hence on $N_{r}\left(x_{0}\right)$, they coincide on $N_{r}\left(x_{0}\right) \cap D$. So $x_{0}$ is in Int $C$. That is, Int $C$ is closed in $D$. Since $D$ is connected, Int $C=D$. q.e.d.

We observe that if $\sum a_{n}\left(x-x_{0}\right)^{n}$ is analytic, with radius $r$, and values in $Y$, and if $h_{n}: Y \rightarrow Z$ is a sequence of continuous linear maps of bounded norm, $\left|h_{n}\right| \leqq K$, then $\sum h_{n} \circ a_{n}\left(x-x_{0}\right)^{n}$ is analytic and has radius $r$ (values in $Z$ ).

THEOREM. If $X$ and $Y$ are topologically isomorphic Banach spaces, $T(X: Y)$ the subspace of $L(X: Y)$ of topological isomorphisms, the inversion map $\iota: T(X: Y) \rightarrow T(Y: X) \subset L(Y: X)$ is an analytic diffeomorphism.

Proof. $T(X: Y)$ is open in $L(X: Y)$, and nonempty by supposition. In [1] or [5] $\iota$ is shown to be a $C^{\infty}$ diffeomorphism. Virtually the same 
proof shows analyticity: let $g \in T(X: Y)$ be fixed, and $f$ in $L(X: Y)$, $\exists|f-g|<1 /\left|g^{-1}\right|$. Then $\left|(f-g) g^{-1}\right|<1$, so $1+(f-g) g^{-1}$ is a topological automorphism of $Y$, so $\left(1+(f-g) g^{-1}\right) g=f$ is a topological isomorphism of $X$ on $Y$. Its inverse is $f^{-1}=g^{-1}\left(1+(f-g) g^{-1}\right)^{-1}=g^{-1}$ $\sum_{n=0}^{\infty}(-1)^{n}\left((f-g) g^{-1}\right)^{n}=\sum_{n=0}^{\infty}(-1)^{n} g^{-1}\left((f-g) g^{-1}\right)^{n}$. But the series $\sum|f-g|^{n}\left|g^{-1}\right|{ }^{n+1}$ converges for $|f-g|<1 /\left|g^{-1}\right|$. q.e.d.

We assume the Inverse Function Theorem as proved in [5] for diffeomorphisms of class $C^{n}, n=1, \cdots, \infty$ and extend it here to analytic diffeomorphisms.

THEOREM. If $f: D \rightarrow Y$ is an analytic diffeomorphism, then $f^{-1}: f(D)$ $\rightarrow D$ is analytic.

Proof. We may suppose $0 \in D$, and $f(0)=0, y=f(x)=a_{1} x+a_{2} x^{2}$ $+\cdots$. Then $f^{\prime}(0)=a_{1}$ is a topological isomorphism. Multiplying by $f^{\prime}(0)^{-1}$, we may suppose $a_{1}=1=$ identity, i.e. $y=x+a_{2} x^{2}+\cdots$ If $x=f^{-1}(y)$ (which is $C^{\infty}$ by the Inverse Function Theorem in the differentiable case) is analytic at 0 , then $x=y+b_{2} y^{2}+\cdots$ and $\sum\left|b_{k}\right||y|^{k}$ converges for $|y|<r$ for some $r>0$, and the coefficients may be determined by recursion (much as in the scalar case); for we can substitute one series in the other, and get $b_{2}=-a_{2}$, etc. The remainder of the proof now follows the classic one of Cauchy, see Knopp [4, pp. 186-190], where one must substitute however our vector series $y=x+a_{2} x^{2}+\cdots$ and $x=y+b_{2} y^{2}+\cdots$ for his real (or complex) series, but otherwise copy that proof; in particular, use the real series there, $y=x-\alpha_{2} x^{2}-\alpha_{3} x^{3}-\cdots$ and $x=y+\beta_{2} y^{2}+\cdots$ ( $x, y$ and coefficients all real now); these are shown to be invertible and guarantee that of ours. q.e.d.

Observe that the proof assures the following more general result: if $f$ is of class $C^{1}$, and analytic at $x_{0}$, and $f^{\prime}\left(x_{0}\right)$ is a topological isomorphism of $X$ on $Y$, then $f$ is a local diffeomorphism at $x_{0}$, whose inverse (existing near $f\left(x_{0}\right)$ ) is analytic at $f\left(x_{0}\right)$.

The Implicit Function Theorem follows easily from the Inverse Function Theorem in standard fashion, without changing any details except that in the assumption of class $C^{n}, n$ may be $a$ (=analytic). We suppose the reader familiar with the proof and omit it:

Implicit Function Theorem. Let $X, Y, Z$ be Banach spaces, $A$ open in $X \times Y$ and $(n=1, \cdots, \infty, a)$ (1) $f \in C^{n}(A: Z)$; (2) $f\left(x_{0}, y_{0}\right)$ $=0$; (3) $D_{2} f\left(x_{0}, y_{0}\right)$ a topological isomorphism of $Y$ on $Z$. Then:

(a) (Existence) There is an open neighborhood of $x_{0}, N\left(x_{0}\right)$, in $X$, and precisely one continuous map $g: N\left(x_{0}\right) \rightarrow Y \ni g\left(x_{0}\right)=y_{0},(x, g(x))$ $\in A$, and $f(x, g(x))=0$;

(b) $g \in C^{n}\left(N\left(x_{0}\right): Y\right)$; 
(c) (Implicit Differentiation) $g^{\prime}(x)=-D_{2} f(x, g(x))^{-1} \circ D_{1} f(x, g(x))$.

We conclude by extending several standard results of complex variable theory, so assume now that $X$ and $Y$ are complex Banach spaces. Our proofs are minor modifications of standard ones for the cases at hand.

Maximum Modulus Theorem. If $D$ is a region in $X$, and $f: D \rightarrow Y$ is analytic, and $f(x)$ (or only $|f(x)|$ ) is not constant, then $|f(x)|$ has no maximum in $D$.

Proof. We assume the result when $X$ and $Y$ are both the complex numbers. Next assume only that $X$ is the complex numbers, and that the assertion is false. Then there is a point $x_{0}$ such that $|f(x)|$ $\leqq\left|f\left(x_{0}\right)\right|$. By Hahn-Banach, let $h$ be a continuous linear form on $Y$ of norm 1 , such that $h\left(f\left(x_{0}\right)\right)=\left|f\left(x_{0}\right)\right|$ (>0, since $f(x)$ is not constant). But $|h f(x)| \leqq|f(x)| \leqq\left|f\left(x_{0}\right)\right|=\left|h f\left(x_{0}\right)\right|$. So $h f$ is constant on $D$, so $=\left|f\left(x_{0}\right)\right|$ on $D$. But $|h f(x)| \leqq|f(x)|$ always, and $|f(x)|<\left|f\left(x_{0}\right)\right|$ at some points $x \Rightarrow|h f(x)|<\left|h f\left(x_{0}\right)\right|$ for some points $x$. Contradiction. So the assertion holds when $X$ is the complex numbers. For general $X$, let $g(t)=f\left(x_{0}+t\left(x-x_{0}\right)\right), t$ complex, and assume $|f(x)| \leqq\left|f\left(x_{0}\right)\right|$, all $x$ in $D . g$ is analytic in $t$ for $|t|<1+r$ for small enough $r>0$, if $x$ is taken close enough to $x_{0}$. Then $|g(t)| \leqq\left|f\left(x_{0}\right)\right|=|g(0)| \Rightarrow g$ constant. So $g(1)=g(0)$, i.e. $f(x)=f\left(x_{0}\right)$. Since $x$ is arbitrary near $x_{0}, f$ is constant near $x_{0}$, hence on $D$. q.e.d.

Liouville's TheOREM. $A$ bounded entire function is constant.

Proof. We assume the theorem when domain and range are the complex numbers. Suppose then $f: X \rightarrow Y$ is bounded, analytic on all $X$. Let $k(x)=f(x)-f(0)$. Then $k: X \rightarrow Y$ is bounded entire and $k(0)$ $=0$. Let $g(t)=k(t x), t$ complex, and let $h$ be any continuous linear form on $Y$. Then $h g(t)$ is bounded entire, hence constant 0 since its value at 0 is 0 . But $|g(t)|=\sup _{|h|-1}|h g(t)|=0 \Rightarrow g(t)=0$, hence $g=0$. But $0=g(1)=k(x)$. But $x$ was arbitrary. So $k=0$. So $f(x)=f(0)$. q.e.d.

Theorem (Liouville). If $f: X \rightarrow Y$ is entire, and $|f(x)| \leqq K|x|^{n}$, every $x$, then $f$ is a polynomial of degree at most $n: f(x)=\sum_{k=0}^{n} a_{k} x^{k}$.

Proof. Let $h$ be a continuous linear form on $Y$ and $f(x)=\sum_{k=0}^{\infty} a_{k} x^{k}$; the series $\sum\left|a_{k}\right||x|^{k}$ has positive radius. Then $g(t)=h f(t x)$ $=\sum_{k=0}^{\infty} h a_{k} x^{k} t^{k}, t$ complex, is entire, and $|h f(t x)| \leqq|h| K|x|^{n}|t|^{n}$, so $g(t)$ (by the scalar case) is a polynomial of degree $\leqq n$. That is, $h a_{k} x^{k}=0$, for $k>n$. But $\left|a_{k} x^{k}\right|=\sup _{|h|=1}\left|h a_{k} x^{k}\right|=0 \Rightarrow a_{k} x^{k}=0, k>n$. But $x$ is arbitrary near 0 . So $a_{k}=0, k>n$. q.e.d. 


\section{REFERENCES}

1. J. Dieudonne, Foundations of modern analysis, Academic Press, New York, 1960.

2. N. Dunford and J. T. Schwartz, Linear operators, Part 1, Interscience, New York, 1958.

3. E. Hille and R. S. Phillips, Functional analysis and semi-groups, Amer. Math. Soc. Colloq. Publ. Vol. 31, Amer. Math. Soc., Providence, R. I., 1957.

4. K. Knopp, Theorie und Anwendung der Unendlichen Reihen, 4th ed., Springer, Berlin, 1947.

5. S. Lang, Introduction to differentiable manifolds, Interscience, New York, 1962. 6. L. Loomis, Abstract harmonic analysis, Van Nostrand, New York, 1953.

7. A. E. Taylor, Introduction to functional analysis, John Wiley, New York, 1958. 